\title{
Towards understanding the roles of prohibitins, multi- functional regulator proteins
}

\author{
Anja Winter ${ }^{1,2} \&$ Andreas Hofmann ${ }^{2 *}$ \\ 'ISMB, School of Biological Sciences, The University of Edinburgh, Scotland, UK \\ ${ }^{2}$ Structural Chemistry Program, Eskitis Institute for Cell \& Molecular Therapies, Griffith University, \\ Brisbane, Australia \\ *Correspondence to Andreas Hofmann, Structural Chemistry Program, Eskitis Institute for Cell \& \\ Molecular Therapies, Griffith University, Brisbane Innovation Park, \\ Don Young Road, Brisbane, Qld 4111 , Australia. \\ Tel.: +61-7-3735-6086, Fax: +61-7-3735-6001 \\ Email: a.hofmann@griffith.edu.au \\ Keywords: Molecular modelling, PHB domain, protein-protein interactions, \\ m-AAA-protease, melanogenin
}

Revised version, 25.05.07 


\begin{abstract}
Prohibitins comprise a family of highly conserved ubiquitous eukaryotic proteins that localise to different compartments of the cell. They have been implicated in important cellular processes such as cellular signalling and transcriptional control, apoptosis, cellular senescence, early development of Caenorhabditis elegans and mitochondrial biogenesis.
\end{abstract}

In yeast, mammals and C. elegans there exist at least two homologous prohibitin proteins (yeast: PHB1, PHB2; human: BAP32, BAP37), which assemble into high molecular weight complexes of about 1.2 $\mathrm{MDa}$ in the inner mitochondrial membrane. Experimentally determined structural information about these proteins has been elusive for a long time. Recently, however, the biogenesis and architecture of the yeast prohibitin complex has been analysed and yielded ring-shaped structures as visualised by single particle electron microscopy.

Structural details at atomic level remain to be determined, but a first step into this direction is provided by modelling approaches. Prohibitins consist of three domains, an N-terminal transmembrane helix, a middle (PHB) domain and a C-terminal coiled coil domain. The PHB domain is the landmark feature within the super-family of SPFH (stomatin / prohibitin / flotillin / HfIK/C) domain proteins. The recently determined NMR structure of mouse flotillin-2 provides a first access to structural details of prohibitins.

While the first functional role attributed to prohibitins was the regulation of cellular senescence, DNA transcription and tumour cell growth, there is recent evidence that they also can act as markers for adipose tissue. In a mouse model, an apoptotic peptide targeted at prohibitin was successful in reversing obesity. An extracellular complex containing both BAP32 and BAP37 was found to bind to the Vi capsular polysaccharide, first identified as a virulence antigen of Salmonella typhi, suggesting a key role for both proteins in infection with S. typhi. Furthermore, the interaction of prohibitin with compounds activating melanin production has placed these proteins at a central position in melanogenesis, and further implicates mitochondria in signalling pathways of the pigmentation process. Accumulating evidence suggests that prohibitins are implicated in mitochondrial, age and oxidative stress -related diseases, as well as in immunity and inflammation, cancer and cancer-like diseases, obesity, and drug resistance.

The complementary interplay between structural and chemical biology will provide important insights into the molecular mechanisms of prohibitins and, more generally, the functions of mitochondria in living cells.

This review discusses the current state of knowledge about prohibitins, and provides a vision for further developments in the field of these eminently important proteins. 


\section{Introduction}

Prohibitins comprise a family of conserved ubiquitous eukaryotic proteins [1]. They have been implicated in important cellular processes and have been found in different cellular compartments, including mitochondria. Prohibitin 1 cDNA was first isolated by hybridisation to RNA in rat liver and the protein was proposed to be an inhibitor of cellular proliferation [2]. Inhibition of DNA synthesis was observed upon microinjection of prohibitin 1 mRNA into human fibroblasts [3], an effect that was subsequently found to be a feature of the $3^{\prime}$-untranslated region of prohibitin 1 [4].

While prohibitin 1 is usually referred to as $\mathrm{Phbl}$, its human orthologue is known as B-cell receptor associated protein 32 (BAP32). The related protein prohibitin 2 (Phb2) is also known as prohibitone [5], and the human orthologue is known as BAP37 which is identical to a protein earlier identified as repressor of estrogen receptor action (REA) [6]. Prohibitin orthologues have also been identified in other mammals, Drosophila, plants and yeast. In yeast, prohibitin 1 is referred to as PHB1, whereas prohibitone is called PHB2.

Prohibitins belong to a larger super family of proteins, called the stomatin/prohibitin/flotillin/HfIK/C (SPFH) super family, that share an evolutionary conserved domain, the SPFH, or PHB domain. Members of this protein family are membrane-associated and implicated in cellular processes concerned with protein turnover [7], senescence [2] and proliferation control [2, 8, 9]. They can be found in higher eukaryotes (prohibitin, stomatin, podocin), as well as in lower eukaryotes and prokaryotes (vacuolin A, vacuolin B, HfIK, HfIC, unc-1, unc-24, mec-2). The PHB domain is conserved in proteins that are ubiquitously expressed such as flotillin, as well as in proteins that show extremely restricted expression like the stomatin-related olfactory protein (SROP) whose expression is constrained to olfactory sensory neurons [10]. The evolutionary significance of this domain is unclear but its prokaryotic conservation suggests that it is indeed a primordial motif likely to perform an important cellular function.

\section{Cellular localisation of prohibitins}

Prohibitins have been found in several intra- as well as extra-cellular locations. In early studies, prohibitins were nearly exclusively found to localise to mitochondria, but later studies reported prohibitins to be localised also in the nucleus and the cytosol, as well as at the cell surface. While it has been suggested that the $\mathrm{N}$-terminal sequences of PHB-domain containing proteins are responsible for subcellular targeting [11], it remains unclear which mechanisms and what signals are used to transport prohibitins to different cell compartments.

With immunohistochemical methods and subcellular fractionation, prohibitins have been found most abundantly in mitochondria [12-15], where they form ring-shaped high molecular weight complexes in the inner mitochondrial membrane [16].

Using differential immunisation, BAP32 and BAP37 have been detected in the human circulation system, with considerably higher levels in cancer patients [17]. The implications of extra-cellular 
prohibitins remain to be elucidated, but BAP32 has recently been linked to complement activation since it interacts with complement component C3 [18]. Most likely, the externalisation of prohibitins happens by release from lipid vesicles rather than protein translocation using signal peptides [19] which might also be the mechanism of prohibitin transport to the endothelium.

Using mouse and human tissues, an immunohistochemical study revealed that prohibitin 1/BAP32 is present in the vasculature of white adipose tissue [20], and may therefore be used as a target for apoptosis inducing drugs to fight obesity. Cell surface -associated BAP32 and BAP37 have also been shown to interact with Vi capsular polysaccharide of Salmonella typhi [21] in the intestinal epithelium. Phb1/BAP32 [22-25] and Phb2/BAP37 [26, 27] were repeatedly shown to be present in the nucleus. BAP32 possesses a nuclear export signal and, in breast cancer cells, translocates to the cytoplasm upon apoptotic stimulation [24, 28, 29]. Recently, prohibitin 2 has been shown to translocate from the mitochondria to the nucleus in the presence of estrogen receptor alpha and estradiol, suggesting a role of this protein in coupling of mitochondrial-nuclear interactions [30].

Both proteins were also found to be associated with cytoskeletal proteins [31], and the association with annexin A2 provides a link of prohibitins to lipid rafts. This notion is further supported by the discovery of the PHB-domain containing proteins erlin-1 and erlin-2 in lipid rafts of the endoplasmic reticulum [11], and the association of lipid rafts with BAP32 and BAP37 on the cell surface [21]. Notably, for podocin and mec-2, the palmitoylated PHB domains have been proven to be essential for the ability to bind cholesterol [32].

\section{Topology and structure of prohibitins}

Topologically, prohibitins can be dissected into three domains: an $\mathrm{N}$-terminal membrane-anchoring $\alpha$ helix, the middle or prohibitin homology (PHB) domain, and the C-terminal coiled coil region. In the absence of atomic details of prohibitin structures, homology models for the PHB domains of both human prohibitins have been generated [33] based on the NMR structure of the related flotillin-2 (PDB accession number 1 win). The domain adopts a slightly elongated globular shape where the two antiparallel $\alpha$-helices pack against a three-stranded anti-parallel $\beta$-sheet. A comparison of the surface properties of the prohibitin models and flotillin-2 reveals interesting differences with the PHB domain of flotillin-2 being predominantly negatively charged. The surface of BAP32, in contrast, presents a mixed arrangement of electropositive and electronegative patches all over the PHB domain, and with BAP37 an almost exclusively electropositive surface potential is observed in the PHB domain. Figure 1 shows representations of the secondary structure elements and surfaces of the modelled PHB domains of BAP32 and BAP37 in combination with their modelled N-terminal helices [33] embedded in the inner mitochondrial membrane. 
Prohibitins show a limited sequence similarity to chaperonins of the GroEL/Hsp60-class. Since prohibitins were found to interact with newly synthesised mitochondrial translation products such as cytochrome c oxidase, they have been proposed to function as a chaperoning "holdase" during the assembly of the respiratory chain [12]. Incidentally, the architecture of the membrane-bound yeast prohibitin complex that has been analysed recently by single particle electron microscopy yielded ringshaped structures with outer dimensions of about $270 \times 200 \AA$ [16] and is thus reminiscent of chaperonins that provide a sequestered environment within cylindrical structures. However, compared to GroEL with a diameter of $150 \AA$, the prohibitin ring is substantially larger. This prompted Langer and colleagues to suggest a scaffolding function of the prohibitin complex that may ensure the organisation and integrity of the inner mitochondrial membrane. This role would also agree with the functional interaction of prohibitins with various mitochondrial proteins reflecting the proposed role of prohibitins for the maintenance of mitochondrial morphology [16].

The ring-like structures of membrane-bound prohibitins imply a topology where prohibitin 1 and 2 associate by an interaction interface that may be formed by the PHB domain. A model of the PHB domain dimer of BAP32:BAP37 has been constructed based on earlier crosslinking experiments [33, 34]. While molecular details remain to be clarified, the ring-like structures of both prohibitins confirm earlier findings of both proteins forming a tight complex and thus stabilising each other $[12,35,36]$. In contrast, Bacher and coworkers reported that both isolated prohibitins, BAP32 and BAP37, preferably form homotetramers as well as calcium-independent heteromeric complexes with affinities of $10^{-7} \mathrm{M}$ [31]. Potentially, BAP32 could form dithioether-based dimers via its cysteine residue in position 69 (see Figure 2) and, intriguingly, is substrate of the transglutaminase 2, a disulphide isomerase [37]. This finding also poses the question of the importance of prohibitin disulphide RedOx chemistry and fold stability for its function. However, since BAP37 does not have any cysteine residue, the BAP32:BAP37 interactions cannot involve dithioether functionalities.

Apart from their oligomeric species, prohibitins 1 and 2 have also been found to interact with target proteins in their monomeric states. In the nucleus, for instance, prohibitin 1 (BAP32) was found to interact with p53 [24], Rb [23, 38], and E2F [39]. Prohibitin 2 (BAP37) was reported to bind to estrogen receptor $(E R)[6,30]$ and $A k t ~[27]$. It remains to be clarified under which circumstances the proteins exist in the respective oligomeric states. Most likely, occurrence of the monomeric state in non-membranebound states would require binding of stabilising proteins to mask the hydrophobic $\mathrm{N}$-terminal domains [33].

The wide range of interactions of prohibitins with other proteins might not only result from different quaternary structures, but also post-translational modifications of the proteins enabling them to carry out various functions in the cell. Phosphorylated species of prohibitins were found in 2D-gel 
electrophoresis experiments in plants [40], and it was also shown that purified JNK1 successfully phosphorylates prohibitin in vitro [39]. While phosphorylation of yeast prohibitin 2 by Akt could not be confirmed despite the presence of a consensus Akt phosphorylation site in prohibitin 2 (86-RPRKIS-91), an interaction of both proteins could be verified in co-immunoprecipitation assays and yeast two-hybrid screens [27]. Residues 120-232 on prohibitin 2 were identified as interaction interface (see figure 2), indicating that it might belong to the class of Akt-binding proteins that are not substrates for the kinase (see next section).

Examination of our proposed PHB domain dimer model of BAP32 and BAP37 [33] shows that most of the three predicted phosphorylation sites (ExPASy proteomics server, www.expasy.ch) in BAP32 (T108, S109, T141) and BAP37 (T194, S190, S110) are surface-accessible (Figure 2). Importantly, some phosphorylation sites are positioned in interaction interfaces to other proteins and might thus be involved in modulation of binding properties to target proteins.

Another post-translational modification might happen by lipo-conjugation of the PHB domain as observed with podocin and mec-2, two PHB domain -containing proteins [32]. As palmitoylation occurs at accessible cysteine residues, only BAP32 (one cysteine at position 69; see Figure 2), but not BAP37 (no cysteine) is anticipated to possess this feature. Based on a preliminary secondary structure alignment of BAP32 with podocin and mec-2, Cys69 seems to be located in a similar position like the conjugated cysteine in podocin/mec-2.

\section{Prohibitins as regulators of gene expression}

Several studies have identified prohibitins as cell cycle regulators, although the detailed mechanisms remain to be clarified $[18,41]$. There is accumulative evidence of prohibitins acting as transcription regulators. Prohibitin 1 interacts with checkpoint molecules of the cell cycle, such as p53 [24] and Rb $[23,38]$, and with the transcription factor E2F [39]. The reported interaction sites of Rb and E2F with prohibitin 1/BAP32 can be mapped on the modelled BAP32:BAP37 dimer unit as illustrated in Figure 2. The region on prohibitin 1 C-terminal to the PHB domain (residues 185 to 214) is required for repression of E2F activity in addition to the Rb-binding domain [39]. The transcription of multiple genes has been found to be modulated by increased cellular levels of prohibitin in transfection experiments, and, accordingly, prohibitin has been implicated in cell cycle regulation [23, 24, 39].

The repressor function of BAP32 with respect to E2F-mediated transcription appears to utilise molecular mediators and signalling pathways different from the Rb pathway [41]. BAP32 recruits co-repressors $\mathrm{HPl} \gamma$ and $\mathrm{Brg} 1 / \mathrm{Brm}$ to repress E2F-mediated transcriptional activity [42-44]. Breast cancer cells treated with estrogen antagonist showed an enhanced association between Brgl/Brm and prohibitin [43, 44].

A similar observation has been reported for the anti-proliferative actions of vitamin D [45]. Cellular levels of BAP32 were found to be increased by vitamin D treatment of breast cancer cell lines subsequently leading to growth inhibition. While a binding site for the vitamin $D$ receptor/retinoid $X$ 
receptor has been predicted in the promoter region of BAP32, direct interactions at protein level remain to be clarified.

The $\mathrm{Rb}$ binding region on BAP32 spans residues 74-116, where three of four point mutations of prohibitin are found in sporadic breast cancer cells [38, 46, 47]. Interestingly, those residues span the anti-parallel $\beta$-sheet of BAP32, which is proposed not to interact with its homologue, BAP37, and would thus be potentially available for interactions with other target proteins. Further research in this area might give clues to the nature of this interaction and thus provide insights in the mechanism responsible for E2F repression.

BAP37, originally known as REA, is a repressor of estradiol-dependent transcription $[6,30]$ and binds directly to the estrogen receptor (ER) in the presence of its ligand estradiol. While BAP37 possesses the common ER-binding motif L-X-X-L-L N-terminal of its PHB domain, residues 175-198 are required for binding to ER [48] (see Figure 2). The L-X-X-L-L motif is suggested to be required for its repressive activity due to inhibiting ER co-activation by SRC-1.

Interestingly, BAP32 was also shown to have a repressive effect on androgen receptor (AR)-mediated transcription and androgen-dependent cell growth without an apparent direct interaction with AR [49].

In the nucleus, Akt has been shown to stimulate the transcription factor families of MyoD and MEF2 indirectly during muscle differentiation. This action was specifically repressed by prohibitin 2, possibly with the help of co-activators such as histone deacetylases. Prohibitin 2 might thus act as a miogenic repressor by competing with Akt to prevent its interaction with MyoD and MEF2 [27].

One can thus speculate that a complex consisting of both prohibitins acts as a mediator between $\mathrm{Rb}$ and E2F (interacting with BAP32) on the one hand, and ER receptor (interacting with BAP37) on the other. Furthermore, these functions might be activated by phosphorylation since some phosphorylation sites are situated in the proposed binding sites of Rb/E2F (see Figure 2).

\section{Prohibitins in cancer}

Early work on BAP32 suggested that the protein is a tumour suppressor, because a growth arrest was found in HeLa cells after microinjection of prohibitin transcripts [3]. It was shown later that this property actually resides in the $3^{\prime}$-UTR of BAP32 mRNA and the ability to inhibit cell cycle activity is restricted to normal cells and cells of the so-called group B immortal complementation group [12]. A polymorphism in the $3^{\prime}$-UTR of BAP32 has been identified as a risk modifier in breast cancer in the presence of BRCA1 mutation [12]. In a screening for somatic mutations in 23 breast cancers, four mutations leading to amino acid changes at positions 88 (Val $\rightarrow$ Ala) and 105 (Arg $\rightarrow$ His), as well as a frame shift mutation leading to a truncation at position 53 [46]. Within an intron, a $C \rightarrow T$ change was observed which may interfere with the splicing process. Interestingly, almost all mutations reported in sporadic breast cancer lie within the Rb-binding domain of prohibitin [46]. 
BAP32 has also been localised to the nuclei of breast cancer cells [24] and the cellular expression of the non-mutated protein is generally up-regulated in tumour cells as compared to normal cells [12, 5053]. BAP32/BAP37 was also found at the cell surface of cultured colorectal tumour cells, and, notably, significant levels of both proteins were found in the serum [17]. It is not entirely clear whether BAP32/BAP37 are intentionally released into the blood or whether this is a direct consequence of the necrosis of tumour cells. In any case, these observations support a probable usability of prohibitins as tumour markers (see also [50]).

The involvement of BAP32 in repression of estrogen-dependent transcription has brought the prohibitin proteins back into focus as potential targets for breast cancer therapy. Estrogen antagonists are the commonly used endocrine therapy in breast cancer, but application is limited due to development of cellular resistance [44]. Further investigation of the prohibitin-E2F interactions and the involvement of estrogen antagonists at the molecular level might lead to development of new drugs in breast cancer therapy. The re-gained attention as potential cancer targets is further deserved because BAP32 was identified as a novel target gene of vitamin $D$, leading to downregulation of proliferation by activation of the vitamin $D$ receptor. The clinical use of vitamin $D$ in cancer therapy and prevention is restricted by its toxic effects at higher concentrations. Recently, BAP32 has been shown to enhance the antiproliferative effects of vitamin D in breast cancer cells [45].

The re-gained interest in prohibitin for cancer treatment is also supported by the observation that interaction of BAP32 with the signalling kinase cRaf is crucial for Ras-mediated activation, as well as membrane targeting of cRaf [54]. BAP32 directly interacts with the kinase and is indispensable for the replacement of 14-3-3 from cRaf by Ras-GTP. Because Ras mutants activating Raf kinases are frequently found in tumours, and BAP32 is a crucial facilitator of this signalling pathway, prohibitin has again become a possible target for therapeutic applications.

Interestingly, a recent study elucidating the mechanisms for occurrence of multidrug resistance in a Caenorhabditis elegans model identified the E130K mutation in prohibitin 2 to be responsible for drug resistance of drugs binding to tubulin and camptothecin [55]. The location of Glu130 in the region of prohibitin 2 binding to $A k t, M y o D$ and $\alpha$-actinin (see table 1) might indicate the direction of further studies to elucidate the molecular mechanism.

\section{The role of prohibitins in apoptosis}

Very early on, the implications of prohibitins for cellular senescence have been emphasised by a number of studies, mainly fuelled by the observation of a shortened replicative life span of an yeast phenotype with disrupted prohibitin $[35,36]$. While disruption of prohibitin genes in S. cerevisiae does not result in a lethal phenotype [35], the deletion of a prohibitin homologue in D. melanogaster [56] and C. elegans [57] is lethal during larval development. In a quest for finding drug-regulated genes in osteosarcoma cells, the cytotoxic chemicals cis-platin, methotrexate and doxorubicin were found to 
increase cellular expression of eight genes mainly coding for electron transfer proteins, but to significantly decrease the expression of prohibitin and $\alpha$-actinin [58]. The role of prohibitin in modulation of drug-induced apoptosis was further confirmed by the finding that its overexpression reduced chemosensitivity of the cells by about $50 \%$.

Similar observations have been made with granulosa cells [59] and plant prohibitins. After inducing defense responses with the protein phosphatase inhibitor calyculin A, rice prohibitin 1 becomes hyperphosphorylated in a lesion-mimic mutant that exhibits spontaneous cell death in the leaf $[40,60]$. The down-regulation of expression of petunia prohibitin 1 by virus-induced gene silencing resulted in an acceleration of senescence of the flower [61]. While the same observation was made for prohibitin 1 in tobacco, the suppression of prohibitin 2 resulted in even more severe growth inhibition and apoptosis [62].

The senescent and apoptotic effects of prohibitin downregulation have always been observed to be accompanied by mitochondrial effects. In normal prostate epithelium, the transforming growth factor- $\beta$ (TGF- $\beta$ ) acts as a tumor suppressor via induction of apoptosis and inhibition of cell proliferation. However, in advanced cancer, TGF- $\beta$ signaling is deregulated which leads to promotion of tumor progression and metastasis acquiring anti-apoptotic pathways. Upon treatment with TGF- $\beta$, prohibitin translocates from the nucleus to the cell cytosol and was found strongly associated with the mitochondrial apoptosis-suppressor Bcl-2 [29]. In the yeast phenotype, a defect of the mitochondrial membrane potential has been observed [35], and the mitochondrial segregation from the mother to the daughter cells seems to be the reason for the shortened lifespan [63]. A disruption of mitochondrial biogenesis has also been found in C. elegans mutants [57]. Recent studies in tobacco show that a depletion of both prohibitins leads to a loss of mitochondrial integrity, excessive production of reactive oxygen species, and, subsequently, over-sensitivity to various stresses [62].

As for possible mechanisms, it has been suggested that the downregulation of prohibitin increases E2F acitvity, and thus E2F-mediated transcription which then triggers the apoptotic processes during the cell cycle at the entry into the S-phase [41]. Very recently, BAP37 has been reported to interact with the antiapoptotic protein Hax-1 [30], an integral membrane protein of the outer mitochondrial membrane that is exposed to the intermembrane space. A model has been postulated where Hax-1 is being protected from proteolytic degradation through association with BAP37. Notably, the authors report indications that the interaction of BAP37 with Hax-1 is stronger than the BAP32-BAP37 interaction [30]. It will be interesting to see in future experiments whether specific, individual target proteins exist for both prohibitins.

\section{Implications for mitochondrial functions}

Different stimuli such as UV irradiation, reactive oxygen species, hormones and growth factors can induce apoptosis which is a well-characterised form of programmed cell death [64] that requires the 
action of caspases. Mitochondria play a pivotal role in the signal transduction of apoptosis, since they trigger the activation of caspases by release of cytochrome c, apoptosis-inducing factor and Smac/Diablo in a process called mitochondrial permeability transition which is the main pathway of programmed cell death in animal cells [65-68].

To date, it is known that prohibitin 1 and 2 assemble into hetero-oligomers to yield high-molecular weight complexes of about 1.2 MDa as shown for yeast [69], mammals [12] and C. elegans [57]. For most interactions with prohibitin target proteins, it is not clear whether this requires reorganisation or indeed disassembly of the prohibitin hetero-oligomers. However, prohibitins seem to be able to interact with several target proteins simultaneously [31].

Further weight was put on the notion that high-molecular weight prohibitin complexes play an important role for the integrity of mitochondrial membranes [36] by the finding that prohibitin mutations in yeast are lethal when combined with disruption of the phosphatidylethanolamine (a major component of mitochondrial membranes) biosynthetic pathway [70]. This hypothesis is further supported by the first structural characterisation of membrane-bound prohibitin ring complexes. The findings by Langer's group indicate a direct effect of the complexes on the ultrastructure of the inner mitochondrial membrane, since the ring-like structures have a similar diameter to cristae junctions [16]. In tobacco, prohibitin depletion causes abnormal mitochondria that lack inner cristae and contain fibre-like structures that probably represent disintegrated inner membrane [62]. The phenotype is further characterised by disrupted mitochondrial biogenesis or stimulated mitochondrial degeneration, and a reduction in number and mass of mitochondria in the cell. Aberrant mitochondria lacking the mitochondrial genome were found to accumulate in S. cerevisae when the function of either prohibitin was lost $[36,63]$. A lack of prohibitins was shown to affect mitochondrial distribution and morphology during development of body wall muscle cells of C. elegans [57]. In this context, RNAi-mediated elimination of prohibitin 2 was found to lead to mitochondrial fragmentation in HeLa cells [30].

The importance of prohibitins for programmed cell death might thus be due to their crucial role for mitochondrial integrity as suggested by recent studies in plants $[40,60]$. Since prohibitins seem to protect inner membrane proteins, modifications such as phosphorylation (of BAP32) might change their functional properties or interactions with other proteins and finally lead to cell death. Accordingly, the high-molecular weight complexes of prohibitins with m-AAA protease are of particular interest. AAA protease, as well as the bacterial orthologue $\mathrm{FtsH}$ and other Fts H-like proteases from plants [71, 72], degrades membrane proteins in mitochondria [73] and seems to be regulated by prohibitins. Recently, the PHB domain -containing protein QmcA was identified in E. coli as a factor involved in membrane protein quality control [74]. As concluded from pulldown assays, QmcA interacts with $\mathrm{FtsH}$ to form oligomers. Based on findings from proteolytic accessibility experiments, these authors also suggested that PHB domains might be present on both sides of the membrane. 
Generally, mitochondrial disorders can give rise to a broad spectrum of diseases such as myopathies, loss of hearing and optic neuropathy [75]. Mitochondrial dysfunction has also been implied in inflammatory diseases such as ulcerative colitis, a disorder characterised by chronic inflammation of the colon mucosa [76]. With a proteomics approach, several mitochondrial proteins have been found to be down-regulated in colon mucosa from patients with ulcerative colitis. This phenomenon is accompanied by pathological alterations of the mitochondrial ultrastructure [76].

Metabolic stress caused by imbalances of mitochondrial and nuclear encoded proteins leads to upregulation of BAP32 and BAP37 expression levels [75]. The mitochondrial prohibitin complex was found to bind to mitochondrial translation products of the respiratory chain such as cytochrome $c$ oxidase (COX). Here, it acts as a chaperone to assemble the COX complex [12], thus exerting important functions in maintaining a healthy protein population in mitochondria.

Disturbances in mitochondrial function might be common in aged population and probably contribute to age-related diseases, such as Type 2 Diabetes [41]. In ageing mitochondria, oxidative stress leads to accumulation of superoxide, which could lead to the promotion of type 2 diabetes [77]. One might speculate that prohibitins could play a role in this process, especially when considering that prohibitin depletion leads to an excessive production of reactive oxygen species in plants [62]. Furthermore, overexpression of prohibitin increases the expression of glutathione-S-transferase $\pi$ and protects from accumulation of reactive oxygen metabolites, as well as increased permeability induced by oxidative stress in intestinal epithelial cells. As such, prohibitin might act as a cellular defense against oxidant injury and thus be an interesting target for tissue injury and inflammatory diseases such as bowel and Crohn's disease [78].

This link deserves further attention, since the addition of BAP32 to cultured adipocytes has been reported to result in inhibition of mitochondrial pyruvate carboxylase and subsequent suppression of glucose and fatty acid oxidation [79].

With a chemical genetics approach searching for pigment-enhancing chemicals, melanogenin has been found to enhance melanin production in melanocytes and to bind specifically to prohibitin [80]. A sufficient pigment production in melanocytes is important for the organism, since UV irradiation can cause severe damage to the cell and finally induce apoptosis through activation of the mitochondrial permeability transition. The damage is possibly caused by favouring the opening of the mitochondrial permeability transition pore. Based on further RNA interference experiments, it has been proposed that prohibitin plays a functional role in melanin induction, since neither the melanin induction agent isobutylmethylxanthine nor melanogenin were able to induce melanin production when prohibitin expression was silenced [80]. Not only opens this finding another avenue for potential therapeutic applications of compounds targeting prohibitin, but it also demonstrates the possibilities of chemical biology approaches to probe organelle functions. 


\section{Prohibitin interactions with cytoskeletal proteins}

Employing an yeast two-hybrid screen, annexin A2 and $\alpha$-actinin have been identified as binding partners for BAP32 and BAP37 [31]. Both proteins are able to bind to various target proteins in a calcium-dependent or a calcium-independent fashion. Annexin A2 is also able to bind to acidic phospholipid membranes in a calcium-regulated fashion.

Truncation experiments by Bacher and colleagues [31] show that for binding to annexin A2, full-length BAP32 or BAP37 is required. The first $100 \mathrm{~N}$-terminal residues of BAP32 displayed a strong binding to $\alpha$-actinin, but in BAP37 the binding site for $\alpha$-actinin seems to reside in the $\mathrm{C}$-terminal domain. While each prohibitin was able to bind to each target protein, the stronger affinities were observed between BAP32 and annexin A2, and BAP37 and $\alpha$-actinin, respectively [31]. The fact that these interactions are calcium-mediated does not automatically suggest a 'sandwich' role for the metal ion, since both target proteins are known to possess calcium-dependent allosteric mechanisms [81].

Notably, prohibitins can interact with several target proteins simultaneously which might also explain the rather moderate in vitro binding affinities determined so far. Homo- and hetero-oligomerisation, as well as ligand interactions probably stabilise the overall complex and thus would increase the affinity constants.

\section{Extra-cellular functions of prohibitins}

In an attempt to find peptide motifs that specifically target white tissue, the motif CKGGRAKDC was specifically localised to and internalised by blood vessels of subcutaneous and peritoneal white fat in mice [20]. The peptide interacts with prohibitin 1 at the protein level which further supports the notion that prohibitins, probably as part of higher order complexes, are present as surface receptors of certain cell types, specifically in endothelial white adipose tissue. The study by these authors [20] went even further in showing that conjugation of a pro-apoptotic peptide to the CKGGRAKDC motif was successful in reversing obesity in the mouse model.

Salmonella typhi is a pathogen that enters the human body by intake of contaminated food or water causing typhoid fever [82]. After intake, the bacterium is translocated to the intestine and subsequently distributed through the reticuloendothelial system. To date, the host-pathogen interactions of Salmonella remain incompletely characterised, but the $\mathrm{Vi}$ capsular polysaccharide had been identified as a major virulence factor [83]. Vi is currently one of the most efficient available vaccines for use in humans [84]. Using a human model intestinal epithelium cell line, the polysaccharide has been shown to interact with a specific cell surface -associated recognition complex that contains BAP32 and BAP37 [21]. Possibly by activating the MAP kinase pathway through the BAP32/BAP37 cell surface complex, Vi reduces an early 
inflammatory response of the host organism $[39,85]$. The role of prohibitins in this context could go beyond extra-cellular recognition, and include a possible participation in intracellular signalling.

Recently, BAP32, but not BAP37, has been shown to circulate in the serum and to exist in a complex with C3, thus being able to enhance complement activation [86]. This ability assigns a potential role to prohibitin in innate immunity which is in agreement with the findings that prohibitins localise to the plasma membrane of human intestinal epithelial cells [21] and lymphocytes [87]. Intriguingly, during infection with S. typhi, serum complement activation is inhibited, if the strains carry the Vi antigen [88]. A link between both findings could be provided by prohibitins, circulating in the serum on the one hand and residing in a cell-surface complex on the other.

\section{Conclusion: Chemical biology of prohibitins}

Prohibitins have first been characterised about 18 years ago, but research on these highly conserved and important proteins has been intensified only in the last six to seven years. By now, the initial focus of prohibitins as new tumour suppressors has been shifted towards detecting intra- and extra-cellular interaction partners, which also diversified the significance of these proteins. The wide range of interaction partners shows that the anticipated fundamental role of prohibitins within the cell has not only been confirmed, but broadened. The involvement in pivotal cellular processes places prohibitins in the context of mitochondrial, age and oxidative stress -related diseases, as well as in immunity and inflammation, cancer and cancer-like diseases, obesity, and drug resistance. While a wealth of information on prohibitin implications in cellular processes has become available, structurally, these proteins are poorly understood. A major breakthrough in this context was achieved by Langer and colleagues who obtained the first visualisation of a low-resolution structure of membrane-bound prohibitins.

Further efforts will now have to be undertaken to obtain more detailed, atomic structure information about the various molecular states of prohibitins. Structural information and their correlation with available functional data will lead to a deeper understanding of the molecular mechanisms of this protein system and reveal their roles in cellular processes.

Chemical biology and structural chemistry will be able to provide this information. The use of small molecules or protein ligands in these studies will not only lend aid in stabilising this protein system in vitro, but will also allow insights into protein-ligand binding. Moreover, this approach will yield valuable data for evaluation of drug interference with the functions of these proteins. Since prohibitins are implicated in a variety of disorders, this will certainly be a highly beneficial undertaking for finding new drugs to fight those diseases. 


\section{Acknowledgements}

Work in the laboratory of $\mathrm{AH}$ is funded by the European League Against Rheumatism (EULAR), Griffith University, and the Parkinson's Disease Society (UK). AW is the recipient of a scholarship of the Darwin Trust of Edinburgh.

\section{Figure legends}

\section{Figure 1}

Model of a putative membrane-anchored hetero-dimer of BAP32 and BAP37 [33]. The hetero-dimer would be part of a larger oligomer forming ring-like structures in the mitochondrial inner membrane as observed by Langer and colleagues [16]. The dimer model of the N-terminal and PHB domains of BAP32 (orange) and BAP37 (blue) assumes a parallel arrangement of both proteins in the membranebound state. Figure prepared with PyMOL [89].

\section{Figure 2}

Visualisation of functionally important sites on prohibitins. Interaction and modification sites are mapped onto the putative hetero-dimer of BAP32 (orange) and BAP37 (blue). Putative phosphorylation sites are indicated by explicitly drawn residues: T108, S109, T141 (BAP32); S1 10, S190, T194 (BAP37). The interaction site of BAP32 with $\mathrm{Rb}$ is shown in green, the interaction site with E2F is shown in cyan. The putative palmitoylation site at Cys69 is indicated explicitly. The BAP37 interaction site with Akt is coloured in yellow, which also includes the binding site for estrogen receptor (purple). Figure prepared with PyMOL [89]. 
Figure 1

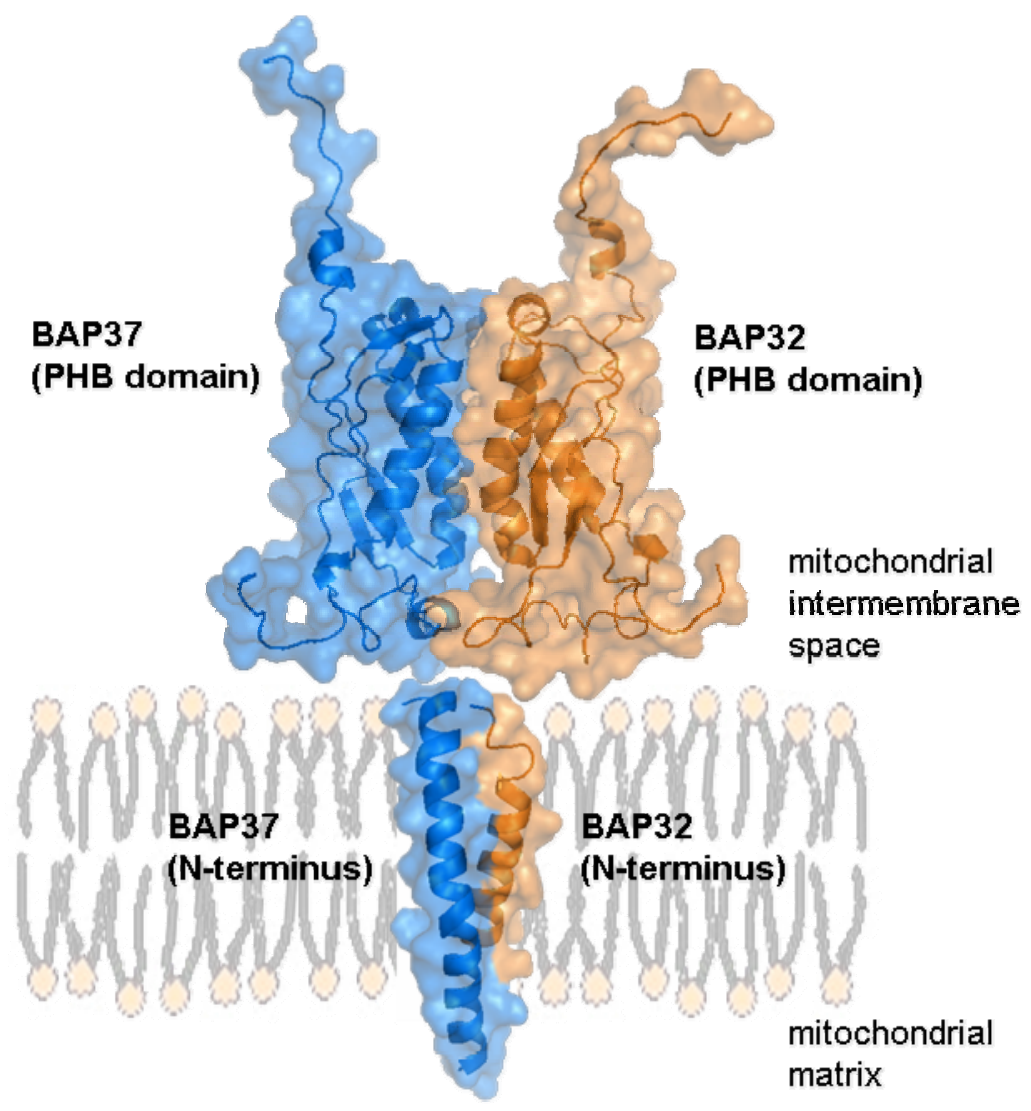


Figure 2

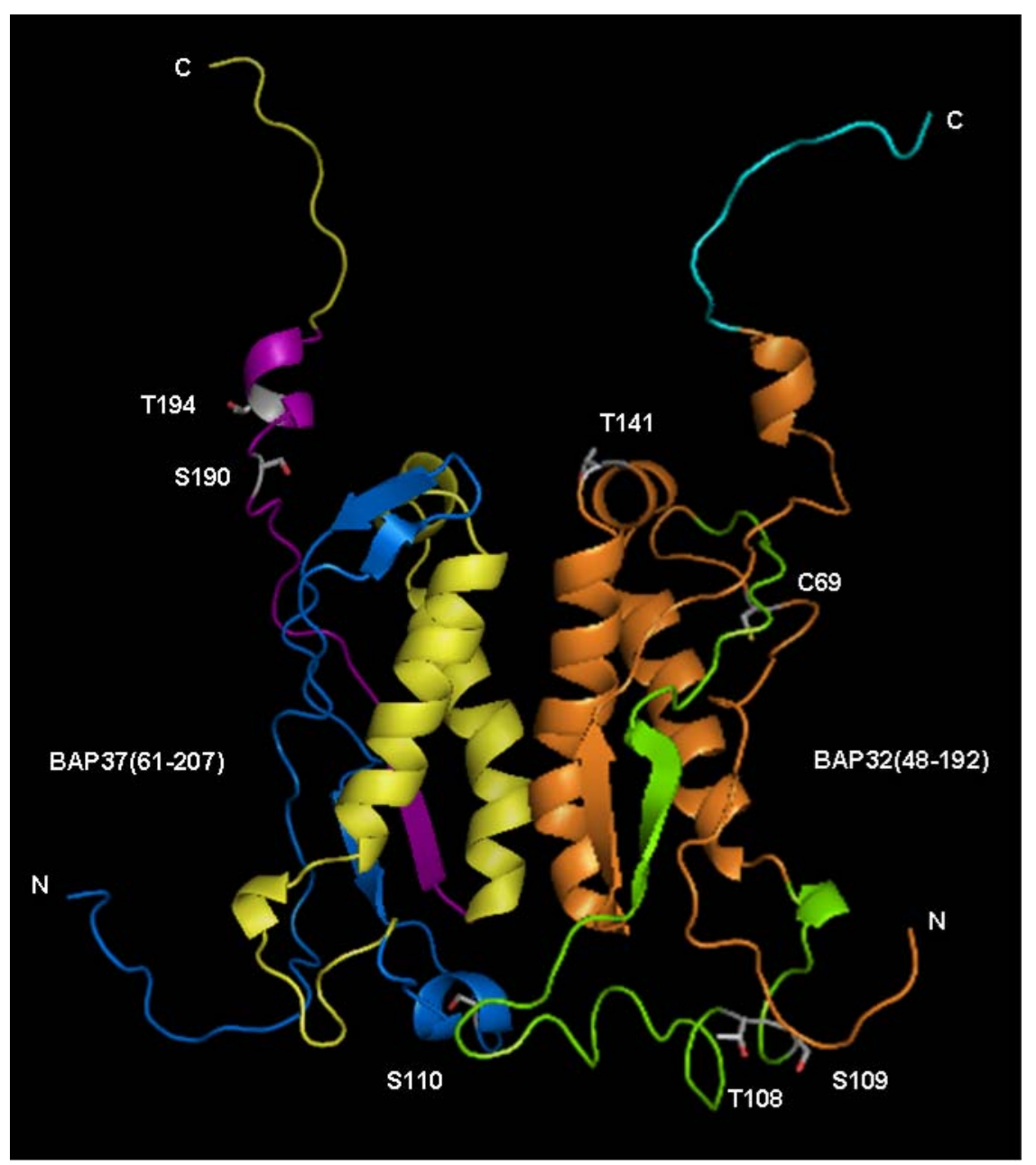




\section{Table 1: Prohibitin-protein interactions}

\begin{tabular}{|c|c|c|c|c|}
\hline Target protein & Prohibitin & Context & Binding sites & Reference \\
\hline Akt & BAP37 & $\begin{array}{l}\text { The protein kinase Akt competitively binds to BAP37 to inhibit binding to } \\
\text { transcription factors (MyoD, MEF2). }\end{array}$ & $\begin{array}{l}\text { BAP37: } 120-232 \\
\text { Akt: } 413-480\end{array}$ & {$[27]$} \\
\hline MyoD & BAP37 & $\begin{array}{l}\text { The transcription factor MyoD belongs to the myogenic regulatory factors } \\
\text { that are important for establishing the myogenic fate of muscle precursor } \\
\text { cells. }\end{array}$ & BAP37: 57-299 & {$[27]$} \\
\hline MEF2 & BAP37 & $\begin{array}{l}\text { MEF2 transcription factors increase the transcription of many muscle-specific } \\
\text { genes. }\end{array}$ & $\begin{array}{l}\text { No direct binding (yeast } \\
\text { two-hybrid) }\end{array}$ & {$[27]$} \\
\hline $\mathrm{Rb}$ & BAP32 & $\begin{array}{l}\text { Binding to } \mathrm{Rb} \text { is necessary for prohibitin to execute its anti-proliferative } \\
\text { action via E2F. }\end{array}$ & BAP32: $74-116$ & {$[6,23,38,39,48]$} \\
\hline E2F & BAP32 & $\begin{array}{l}\text { Repression of E2F caused by prohibitin leads to abolished transcription of } \\
\text { cell-cycle genes like cyclin E and A. }\end{array}$ & BAP32: $185-214$ & {$[38,39,48]$} \\
\hline $\begin{array}{l}\text { Estrogen } \\
\text { receptor (ER) }\end{array}$ & BAP37 & BAP37 dampens response of ER to extra-cellular estradiol stimulation. & BAP37: 175-198 & {$[30,38,39,48]$} \\
\hline Annexin A2 & $\begin{array}{l}\text { BAP32 } \\
\text { BAP37 }\end{array}$ & $\begin{array}{l}\text { Interaction negatively regulated by calcium, association with lipid rafts, } \\
\text { plasma membrane, possible presence in nucleus. }\end{array}$ & $\begin{array}{l}\text { Full-length BAP32 } \\
\text { Full-length BAP37 }\end{array}$ & [31] \\
\hline$\alpha$-actinin & $\begin{array}{l}\text { BAP32 } \\
\text { BAP37 }\end{array}$ & $\begin{array}{l}\text { Interaction negatively regulated by calcium, association with plasma } \\
\text { membrane, interaction with other cytoskeletal proteins, possible } \\
\text { translocation to the nucleus. }\end{array}$ & $\begin{array}{l}\text { BAP32: } 1-99 \\
\text { BAP37: } 118-299\end{array}$ & [31] \\
\hline $\begin{array}{l}\text { VDAC-2, } \\
\text { ANT } 2\end{array}$ & BAP37 & No data available. & & {$[30]$} \\
\hline Hax-1 & BAP37 & BAP37 is involved in the regulation and stability of the anti-apoptotic Hax-1. & $\begin{array}{l}\text { Co-immunoprecipation } \\
\text { of BAP37 and Hax-1 }\end{array}$ & [30] \\
\hline
\end{tabular}




\section{References}

[1] Dell'Orco RT, McClung JK, Jupe ER, et al. Prohibitin and the senescent phenotype. Exp Gerontol 1996; 31:245-52.

[2] McClung JK, Danner DB, Stewart DA, et al. Isolation of a cDNA that hybrid selects antiproliferative mRNA from rat liver. Biochem Biophys Res Comm 1989; 164:1316-22.

[3] Nuell MJ, Stewart DA, Walker L, et al. Prohibitin, an evolutionarily conserved intracellular protein that blocks DNA synthesis in normal fibroblasts and HeLa cells. Mol Cell Biol 1991; 11:1372-81.

[4] Manjeshwar S, Lerner MR, Zang XP, et al. Expression of prohibitin 3' untranslated region suppressor RNA alters morphology and inhibits motility of breast cancer cells. J Mol Histol 2004; 35:639-46.

[5] Lamers MC, Bacher S. Prohibitin and prohibitone, ubiquitous and abundant proteins that are reluctant to reveal their real identity. Int Arch Allergy Immunol 1997; 1 13:146-9.

[6] Montano MM, Ekena K, Delage-Mourroux R, et al. An estrogen receptor-selective coregulator that potentiates the effectiveness of antiestrogens and represses the activity of estrogens. Proc Natl Acad Sci USA 1999; 96:6947-52.

[7] Tavernarakis N, Driscoll M, Kyrpides NC. The SPFH domain: implicated in regulating targeted protein turnover in stomatins and other membrane-associated proteins. Trends Biochem Sci 1999; 24:425-7.

[8] Nuell MJ, McClung JK, Smith JR, et al. Approach to the isolation of antiproliferative genes. Exp Gerontol 1989; 24:469-76.

[9] Roskams AJ, Friedman V, Wood CM, et al. Cell cycle activity and expression of prohibitin mRNA. J Cell Physiol 1993; 157:289-95.

[10] Kobayakawa K, Hayashi R, Morita K, et al. Stomatin-related olfactory protein, SRO, specifically expressed in the murine olfactory sensory neurons. J Neurosci 2002; 22:5931-7.

[11] Browman DT, Resek ME, Zajchowski LD, et al. Erlin-1 and erlin-2 are novel members of the prohibitin family of proteins that define lipid-raft-like domains of the ER. J Cell Sci 2006; 119:3149-60.

[12] Nijtmans LG, de Jong L, Artal Sanz $M$, et al. Prohibitins act as a membrane-bound chaperone for the stabilization of mitochondrial proteins. EMBO J 2000; 19:2444-51.

[13] Santamaria E, Avila MA, Latasa MU, et al. Functional proteomics of nonalcoholic steatohepatitis: mitochondrial proteins as targets of S-adenosylmethionine. Proc Natl Acad Sci 2003; 100:3065-70.

[14] Matsuyama S, Kubo K, Ohashi F, et al. Partial cloning of prohibitin cDNA from canine, feline, bovine, equine, and rabbit liver mRNA by RT-PCR. J Vet Med Sci 1997; 59:201-3.

[15] McClung JK, Jupe ER, Liu XT, et al. Prohibitin: Potential role in senescence, development, and tumor suppression. Exp Gerontol 1995; 30:99-124.

[16] Tatsuta T, Model K, Langer T. Formation of Membrane-bound Ring Complexes by Prohibitins in Mitochondria. Mol Biol Cell 2005; 16:248-59.

[17] Mengwasser J, Piau A, Schlag P, et al. Differential immunization identifies PHB1/PHB2 as blood-borne tumor antigens. Oncogene 2004; 23:7430-5.

[18] Mishra S, Murphy LC, Murphy LJ. The Prohibitins: emerging roles in diverse functions. J Cell Mol Med 2006; 10:353-63.

[19] Brasaemle DL, Dolios G, Shapiro L, et al. Proteomic analysis of proteins associated with lipid droplets of basal and lipolytically-stimulated 3T3-L1 adipocytes. J Biol Chem 2004; 279:46835-42.

[20] Kolonin MG, Saha PK, Chan L, et al. Reversal of obesity by targeted ablation of adipose tissue. Nat Med 2004; 10:625-32.

[21] Sharma A, Qadri A. Vi polysaccharide of Salmonella typhi targets the prohibitin family of molecules in intestinal epithelial cells and suppresses early inflammatory responses. Proc Natl Acad Sci 2004; 101:17492-7.

[22] Thompson WE, Branch A, Whittaker JA, et al. Characterisation of prohibitin in a newly established rat ovarian granulosa cell line. Endocrinol 2001; 142:4076-85. 
[23] Wang S, Fusaro G, Padmanabhan J, et al. Prohibitin co-localizes with $\mathrm{Rb}$ in the nucleus and recruits $\mathrm{N}-\mathrm{CoR}$ and $\mathrm{HDAC} 1$ for transcriptional repression. Oncogene 2002; $21: 8388-96$.

[24] Fusaro G, Dasgupta P, Rastogi $S$, et al. Prohibitin induces the transcriptional activity of p53 and is exported from the nucleus upon apoptotic signaling. J Biol Chem 2003; 278:47853-61.

[25] Gamble SC, Odontiadis M, Waxman J, et al. Androgens target prohibitin to regulate proliferation of prostate cancer cells. Oncogene 2004; 23:2996-3004.

[26] Kurtev V, Margueron R, Kroboth K, et al. Transcriptional regulation by the repressor of estrogen receptor activity via recruitment of histone deacetylases. J Biol Chem 2004; 279:24834-43.

[27] Sun L, Liu L, Yang XJ, et al. Akt binds prohibitin 2 and relieves its repression of MyoD and muscle differentiation. J Cell Sci 2004; 117:3021-9.

[28] Rastogi S, Joshi B, Fusaro G, et al. Camptothecin induces nuclear export of prohibitin preferentially in transformed cells through a CRM-1 dependent mechanism. J Biol Chem 2005.

[29] Zhu B, Fukada K, Zhu H, et al. Prohibitin and Cofilin Are Intracellular Effectors of Transforming Growth Factor $\beta$ Signaling in Human Prostate Cancer Cells. Cancer Res 2006; 66:8640-7.

[30] Kasashima K, Ohta E, Kagawa Y, et al. Mitochondrial functions and estrogen receptordependent nuclear translocation of pleiotropic human prohibitin 2. J Biol Chem 2006; 281:36401-10.

[31] Bacher S, Achatz G, Schmitz ML, et al. Prohibitin and prohibitone are contained in high molecular weight complexes and interact with alpha-actinin and annexin A2. Biochimie 2002; 84:1207-20.

[32] Huber TB, Schermer B, Muller RU, et al. Podocin and MEC-2 bind cholesterol to regulate the activity of associated ion channels. Proc Natl Acad Sci 2006; 103:17079-86.

[33] Winter A, Kamarainen O, Hofmann A. Molecular modeling of prohibitin domains. Proteins 2007.

[34] Back JW, Sanz MA, De Jong L, et al. A structure for the yeast prohibitin complex: Structure prediction and evidence from chemical crosslinking and mass spectrometry. Prot Sci 2002; $11: 2471-8$.

[35] Coates PJ, Jamieson DJ, Smart K, et al. The prohibitin family of mitochondrial proteins regulate replicative lifespan. Curr Biol 1997; 7:607-10.

[36] Berger $\mathrm{KH}$, Yaffe MP. Prohibitin family members interact genetically with mitochondrial inheritance components in Saccharomyces cerevisiae. Mol Cell Biol 1998; 18:4043-52.

[37] Mastroberardino PG, Farrace MG, Viti I, et al. "Tissue" transglutaminase contributes to the formation of disulphide bridges in proteins of mitochondrial respiratory complexes. Biochim Biophys Acta 2006; 1757:1357-65.

[38] Wang S, Nath N, Adlam M, et al. Prohibitin, a potential tumor suppressor, interacts with RB and regulates E2F function. Oncogene 1999; 18:3501-10.

[39] Wang S, Nath N, Fusaro G, et al. Rb and prohibitin target distinct regions of E2Fl for repression and respond to different upstream signals. Mol Cell Biol 1999; 19:7447-60.

[40] Takahashi A, Kawasaki T, Wong HL, et al. Hyperphosphorylation of a mitochondrial protein, prohibitin, is induced by calyculin $A$ in a rice lesion-mimic mutant cdr1. Plant Physiol 2003; 132:1861-9.

[41] Mishra S, Murphy LC, Nyomba BL, et al. Prohibitin: a potential target for new therapeutics. Trends Mol Med 2005; 11:192-7.

[42] Rastogi S, Joshi B, Dasgupta $P$, et al. Prohibitin facilitates cellular senescence by recruiting specific corepressors to inhibit E2F target genes. Mol Cell Biol 2006; 26:4161-71.

[43] Wang S, Zhang B, Faller DV. Prohibitin requires Brg-1 and Brm for the repression of E2F and cell growth. EMBO J 2002; 21:3019-28.

[44] Wang S, Zhang B, Faller DV. BRG1/BRM and prohibitin are required for growth suppression by estrogen antagonists. EMBO J 2004; 23:2293-303.

[45] Peng X, Mehta R, Wang $S$, et al. Prohibitin is a novel target gene of vitamin d involved in its antiproliferative action in breast cancer cells. Cancer Res 2006; 66:7361-9.

[46] Sato T, Saito H, Swensen J, et al. The human prohibitin gene located on chromosome 17q21 is mutated in sporadic breast cancer. Cancer Res 1992; 52:1643-6. 
[47] Sato T, Sakamoto T, Takita K, et al. The human prohibitin (PHB) gene family and its somatic mutations in human tumors. Genomics 1993; 17:762-4.

[48] Delage-Mourroux R, Martini PG, Choi I, et al. Analysis of estrogen receptor interaction with a repressor of estrogen receptor activity (REA) and the regulation of estrogen receptor transcriptional activity by REA. J Biol Chem 2000; 275:35848-56.

[49] Gamble SC, Chotai D, Odontiadis M, et al. Prohibitin, a protein downregulated by androgens, represses androgen receptor activity. Oncogene 2007; 26:1757-68.

[50] Wang KJ, Wang RT, Zhang JZ. Identification of tumor markers using two-dimensional electrophoresis in gastric carcinoma. World J Gastroenterol 2004; 10:2179-83.

[51] Asamoto M, Cohen SM. Prohibitin gene is overexpressed but not mutated in rat bladder carcinomas and cell lines. Cancer Lett 1994; 83:201-7.

[52] Shan L. cDNA microarray profiling of rat mammary gland carcinomas induced by 2-amino-1methyl-6-phenylimidazo-4,5- $\beta$-pyridine and 7,12-dimethyl-benz- $\alpha$-anthracene. Carcinogenesis 2002; 23:1561-8.

[53] Dowling $P$, Meleady $P$, Dowd A, et al. Proteomic analysis of isolated membrane fractions from superinvasive cancer cells. Biochim Biophys Acta 2007; 1774:93-101.

[54] Rajalingam K, Wunder C, Brinkmann V, et al. Prohibitin is required for Ras-induced Raf-MEKERK activation and epithelial cell migration. Nat Cell Biol 2005; 7:837-43.

[55] Zubovych I, Doundoulakis T, Harran PG, et al. A missense mutation in Caenorhabditis elegans prohibitin 2 confers an atypical multidrug resistance. Proc Natl Acad Sci 2006; 103:15523-8.

[56] Eveleth DDJ, Marsh JL. Sequence and expression of the Cc gene, a member of the dopa decarboxylase gene cluster of Drosophila: possible translational regulation. Nucleic Acids Res 1986; 14:6169-83.

[57] Artal-Sanz M, Tsang WY, Willems EM, et al. The mitochondrial prohibitin complex is essential for embryonic viability and germline function in Caenorhabditis elegans. J Biol Chem 2003; 278:32091-9.

[58] Fellenberg J, Dechant MJ, Ewerbeck $V$, et al. Identification of drug-regulated genes in osteosarcoma cells. Int J Cancer 2003; 105:636-43.

[59] Chowdhury I, XU W, Stiles JK, et al. Apoptosis of Rat Granulosa Cells After Staurosporine And Serum Withdrawal Is Suppressed by Adenovirus Directed Overexpression of Prohibitin. Endocrinology 2007; 148:206-17.

[60] Takahashi A, Kawasaki T, Henmi K, et al. Lesion mimic mutants of rice with alterations in early signaling events of defense. Plant J 1999; 17:535-45.

[61] Chen JC, Jiang CZ, Reid MS. Silencing a prohibitin alters plant development and senescence. Plant J 2005; 44:16-24.

[62] Ahn CS, Lee JH, Reum Hwang A, et al. Prohibitin is involved in mitochondrial biogenesis in plants. Plant J 2006; 46:658-67.

[63] Piper PW, Jones GW, Bringloe D, et al. The shortened replicative life span of prohibitin mutants of yeast appears to be due to defective mitochondrial segregation in old mother cells. Aging Cell 2002; 1:149-57.

[64] Jacobson MD, Weil M, Raff MC. Programmed cell death in animal development. Cell 1997; 88:347-54.

[65] Green DR, Reed JC. Mitochondria and apoptosis. Science 1998; 281:1309-12.

[66] Susin SA, Lorenzo HK, Zamzami N, et al. Molecular characterisation of mitochondrial apoptosis-inducing factor. Nature 1999; 397:441-6.

[67] Verhagen AM, Ekeert PG, Pakusch $M$, et al. Identification of DIABLO, a mammalian protein that promotes apoptosis by binding to and antagonising IAP proteins. Cell 2000; 102:43-53.

[68] Lam E, Kato N, Lawton M. Programmed cell death, mitochondria and the plant hypersensitive response. Nature 2001; $411: 848-53$.

[69] Steglich G, Neupert W, Langer T. Prohibitins regulate membrane protein degradation by the $m$ AAA protease in mitochondria. Mol Cell Biol 1999; 19:3435-42. 
[70] Birner R, Nebaver R, Schneiter R, et al. Synthetic Lethal Interaction of the Mitochondrial Phosphatidylethanolamine Biosynthetic Machinery with the Prohibitin Complex of Saccharomyces cerevisiae. Mol Biol Cell 2003; 14:370-83.

[71] Adam Z, Adamska I, Nakabayashi K, et al. Chloroplast and mitochondrial proteases in Arabidopsis: a proposed nomenclature. Plant Physiol 2001; 125:1912-8.

[72] Seo S, Okamoto M, Iwai T, et al. Reduced levels of chloroplast FtsH protein in tobacco mosaic virus-infected tobacco leaves accelerate the hypersensitive reaction. Plant Cell 2000; 12:91732.

[73] Leonhard K, Guiard B, Pellecchia G, et al. Membrane protein degradation by AAA proteases in mitochondria: extraction of substrates from either membrane surface. Mol Cell Biol 2000; 5:629-38.

[74] Chiba S, Ito K, Akiyama Y. The Escherichia coli plasma membrane contains two PHB (prohibitin homology) domain protein complexes of opposite orientations. Mol Microbiol 2006; 60:44857.

[75] Nijtmans LG, Artal SM, Grivell LA, et al. The mitochondrial PHB complex: roles in mitochondrial respiratory complex assembly, ageing and degenerative disease. Cell Mol Life Sci 2002; 59:143-55.

[76] Hsieh SY, Shih TC, Yeh CY, et al. Comparative proteomic studies on the pathogenesis of human ulcerative colitis. Proteomics 2006; 6:5322-31.

[77] Lowell BB, Shulman GI. Mitochondrial dysfunction and type 2 diabetes. Science 2005; 307:384-7.

[78] Theiss AL, Idell RD, Srinivasan S, et al. Prohibitin protects against oxidative stress in intestinal epithelial cells. FASEB J 2007; 21:197-206.

[79] Vessal M, Mishra S, Moulik S, et al. Prohibitin attenuates insulin-stimulated glucose and fatty acid oxidation in adipose tissue by inhibition of pyruvate carboxylase. FEBS J 2006; 273:56876.

[80] Snyder JR, Hall A, Ni-Komatsu L, et al. Dissection of melanogenesis with small molecules identifies prohibitin as a regulator. Chem Biol 2005; 12:477-84.

[81] Hofmann A, Huber R. Structural conservation and functional versatility: Allostery as a common annexin feature. In: Bandorowicz-Pikula J, ed. Annexins: Biological importance and annexinrelated pathologies. Georgetown, TX: Landes Bioscience 2003:38-60.

[82] Keusch GT. In: Wilson JD, Braunwald E, Isselbacher KJ, Petersdorf RG, Martin JB, Fauci AS, et al., eds. Harrison's Principal of Internal Medicine. New York: McGraw-Hill 1991:609-13.

[83] Szu SC, Bystricky S. Physical, chemical, antigenic, and immunologic characterization of polygalacturonan, its derivatives, and $\mathrm{Vi}$ antigen from Salmonella typhi. Methods Enzymol 2003; 363:552-67.

[84] Lin FY, Ho VA, Khiem HB, et al. The efficacy of a Salmonella typhi Vi conjugate vaccine in twoto-five-year-old children. N Engl J Med 2001; 344:1263-9.

[85] Hobbie S, Chen LM, Davis RJ, et al. Involvement of mitogen-activated protein kinase pathways in the nuclear responses and cytokine production induced by Salmonella typhimurium in cultured intestinal epithelial cells. J Immunol 1997; 159:5550-9.

[86] Mishra S, Moulik S, Murphy LJ. Prohibitin binds to C3 and enhances complement activation. Mol Immunol 2007; 44:1907-12.

[87] Terashima M, Kim K-M, Adachi $T$, et al. The IgM antigen receptor of B lymphocytes is associated with prohibitin and a prohibitin-related protein. EMBO J 1994; 13:3782-92.

[88] Hornick RB, Greisman SE, Woodward TE, et al. Typhoid fever: pathogenesis and immunologic control. N Engl J Med 1970; 283:686-91.

[89] DeLano WL. The PyMOL Molecular Graphics System. http://wwwpymolorg 2002. 